\title{
Cellular immunophenotypic profile in the splenic compartment during canine visceral leishmaniasis
}

\author{
Alexandre Barbosa Reis ${ }^{a, b, f, *}$, Andréa Teixeira-Carvalho ${ }^{c}$, \\ Rodolfo Cordeiro Giunchetti ${ }^{a}, \mathrm{~d}$, Bruno Mendes Roatt ${ }^{\mathrm{b}}$, Wendel Coura-Vital ${ }^{\mathrm{b}}$, \\ Roney de Carvalho Nicolato ${ }^{\mathrm{a}}$, Denise Silveira-Lemos ${ }^{\mathrm{b}}$, \\ Rodrigo Corrêa-Oliveira ${ }^{\mathrm{e}}$, Olindo de Assis Martins-Filho ${ }^{\mathrm{c}}$ \\ a Programa de Pós-Graduação em Ciências Farmacêuticas, Escola de Farmácia, Universidade Federal de Ouro Preto, Ouro Preto, CEP \\ 35400-000 Minas Gerais, Brasil \\ b Núcleo de Pesquisa em Ciências Biológicas, Universidade Federal de Ouro Preto, Ouro Preto, Minas Gerais CEP $35400-000$, Brasil \\ c Laboratório de Biomarcadores de Diagnóstico e Monitoração, Centro de Pesquisas René Rachou, Fundação Oswaldo Cruz, Belo \\ Horizonte, CEP 21040-360 Minas Gerais, Brasil \\ d Departamento de Morfologia, Universidade Federal de Minas Gerais, Belo Horizonte, CEP 31270-901 Minas Gerais, Brasil \\ e Laboratório de Imunologia Celular e Molecular, Centro de Pesquisas René Rachou, Fundação Oswaldo Cruz, Belo Horizonte, CEP \\ 21040-360 Minas Gerais, Brasil \\ ${ }^{\mathrm{f}}$ Instituto Nacional de Ciência e Tecnologia em Doenças Tropicais, Salvador, Bahia, Brasil
}

\section{A R T I C L E I N F O}

\section{Article history:}

Received 23 June 2013

Received in revised form

30 September 2013

Accepted 22 November 2013

\section{Keywords:}

Canine visceral leishmaniasis

Leishmania infantum

Splenic compartment

Cellular immune response

\begin{abstract}
A B S T R A C T
To determine the role of the spleen in the pathogenesis of canine visceral leishmaniasis (CVL), we analyzed cellular immunophenotypic profiles of 52 dogs naturally infected with Leishmania infantum, clinically classified as follows: asymptomatic dogs-I (AD-I), seronegative/PCR+; asymptomatic dogs-II (AD-II), seropositive/PCR+; oligosymptomatic dogs (OD) and symptomatic dogs (SD). Seven non-infected dogs (CD) were included as a control group. AD-II presented higher levels of CD8+ T splenocytes and lower TCD4+/TCD8+ ratio in comparison with CD. OD and SD showed lower percentages of CD21+ as compared with AD-II. All seropositive dogs presented lower levels of CD45RA+ than CD. Regardless of the stimuli used, the proliferation index from splenocytes in vitro was inversely correlated with clinical status. After LSA stimulation, there was a higher percentage of specific CD8+ T in AD-Il than $\mathrm{CD}$ and non-stimulated culture. In contrast, splenocytes from SD under in vitro LSA stimulation induced decreased MHC-II+ expression in comparison with all groups, and nonstimulated culture. In conclusion, the role of CD8+ T splenocytes seems to be important for an effective immunological response, a hallmark of asymptomatic CVL, whereas the pronounced loss of MHC-II expression upon LSA stimulation is a biomarker of symptomatic CVL.
\end{abstract}

(C) 2013 Elsevier B.V. All rights reserved.

\footnotetext{
* Corresponding author at: Programa de Pós-Graduação em Ciências Farmacêuticas, Escola de Farmácia, Universidade Federal de Ouro Preto, Ouro Preto, CEP 35400-000 Minas Gerais, Brasil. Tel.: +55 2131 35591694; fax: +55 213135591680 .

E-mail addresses: alexreis@nupeb.ufop.br, alexreisufop@gmail.com (A.B. Reis).
}

\section{Introduction}

Visceral leishmaniasis (VL) is a potentially fatal human disease caused by the intracellular protozoan parasite Leishmania infantum (Kaye et al., 2004). Canine visceral leishmaniasis (CVL) represents a problem for both veterinary medicine and public health in approximately 50 countries in various endemic areas of the world (CouraVital et al., 2011b; Desjeux, 2004). CVL is a systemic, 
chronic, and severe disease that is often fatal because no efficacious drugs exist to cure these animals (Baneth et al., 2008; Reis et al., 2006c). According to Mancianti et al. (1988), CVL can be categorized into three distinct clinical forms, based on major clinical features observed in infected dogs: asymptomatic (AD), with no suggestive signs of the disease; oligosymptomatic (OD), with a maximum of three clinical signs; and symptomatic (SD), with typical clinical signs, showing the most severe clinical signs of CVL.

Recently, Coura-Vital et al. (2011a) proposed a new classification scheme for the canine disease according to serological, molecular, and clinical features. The asymptomatic form was divided in two subgroups: asymptomatic dogs I (AD-I), with negative serological tests for Leishmania but positive molecular results, and asymptomatic dogs II (AD-II), showing positive results for both serology and molecular analyses for L. infantum.

VL is a disease associated with the inability of lymphocytes to activate $M \Phi$ to kill Leishmania (Nylen and Sacks, 2007). Parasites can be found in mononuclear phagocytic cells in the spleen and liver, which are the major affected sites (Kaye et al., 2004; Stanley and Engwerda, 2007). In a progressive disease, cellular immune response are impaired, as indicated by studies showing that peripheral blood mononuclear cells (PBMCs) from affected humans and dogs fail to respond to parasite soluble antigens both in vitro and in vivo (Boggiatto et al., 2010; Goto and Prianti, 2009). On the other hand, a protective immune response (observed in asymptomatic VL) is manifested by a strong proliferative response of PBMCs from affected humans and dogs to Leishmania antigens, and the production of cytokines, such as IFN- $\gamma$ and TNF- $\alpha$, which are required for $\mathrm{M} \Phi$ activation and killing of intracellular parasites (Cabral et al., 1992, 1998; Pinelli et al., 1994, 1999; Seixas Duarte et al., 2008; Strauss-Ayali et al., 2005).

Some studies have shown that the proliferation capability of PBMCs from dogs with CVL was decreased upon both antigenic and mitogenic stimulation (Cabral et al., 1998; Cardoso et al., 2007; Pinelli et al., 1995). However, the influence of the populations and subpopulations of the splenocytes derived from dogs with CVL upon antigenic stimulation is not completely understood. Because the spleen is one of the major organs affected during CVL, and the contribution of the compartmentalized immune response in the genesis of splenomegaly during this infection remains unclear, it is relevant to investigate significant alterations in the phenotypic features from infected dogs, performing a detailed ex vivo and in vitro immunophenotyping of their splenocytes.

\section{Materials and methods}

\subsection{Dogs and experimental design}

All procedures in this study were according to the guidelines set by the Brazilian Animal Experimental College (COBEA). This study was approved by the Ethical Committee for the Use of Experimental Animals at the Universidade Federal de Minas Gerais, Brazil (Protocol no. 020/2007).

Fifty-two mixed-breed adult dogs of both genders (age ranging from 2 to 6 years) were selected. They were maintained in the kennel at the Instituto de Ciências Biológicas, Universidade Federal de Minas Gerais or provided by the Centro de Zoonoses-Belo Horizonte/Minas Gerais, Brazil. The dogs used in this study were stray or domiciled mongrel dogs, selected based on their serological results on IFAT (IFI-Leishmaniose-VisceralCanina-Bio-Manguinhos, Rio de Janeiro, Brazil), which is the gold standard immunological test for diagnosis of CVL. Animals presenting IFAT titers $\geq 1: 40$ were considered positive and included into the infected groups. Animals with IFAT negative at 1:40 were considered non-infected and included as a control group. Positive infection was confirmed by PCR in at least one skin sample (Degrave et al., 1994), and the species of Leishmania responsible for the infection were determined by restriction fragment length polymorphism-PCR (Volpini et al., 2004).

\subsection{Clinical classification}

The dogs were clinically classified according to presence/absence of infection signs serological, and molecular tests: asymptomatic dogs I (AD-I, $n=8)$, with no suggestive signs of disease, negative serology, and PCR+ for Leishmania; asymptomatic dogs II (AD-II, $n=10$ ), with no suggestive signs of disease, positive serology, and PCR+ for Leishmania; oligosymptomatic dogs (OD, $n=11$ ), with a maximum of three clinical signs including opaque bristles, and/or localized alopecia, and/or moderate loss of weight; and symptomatic dogs (SD, $n=16$ ), with characteristic clinical signs of CVL, such as opaque bristles, severe loss of weight, onychogryphosis, cutaneous lesions, apathy, and keratoconjunctivitis, and both groups with positive serology, and PCR+ for Leishmania; and control dogs (CD, $n=7)$, classified according to negative serological and PCR- for Leishmania.

\subsection{Spleen sample collection}

The collection of spleen specimens was carried out after euthanasia of the dogs with a barbituric anesthesia (Thiopental at $30 \mathrm{mg} / \mathrm{kg}$ body weight). Spleen fragments ( $5 \mathrm{~mm}$ ) were stored on ice up to $12 \mathrm{~h}$ in a Petri dish in sterile RPMI-1640 (Gibco, Grand Island, NY, USA) prior to use in ex vivo and in vitro procedures. The tissue was minced in a tissue grinder and transferred to $2 \mathrm{~mL}$ of RPMI-1640. The cells suspension was then filtered on stainless steel gauze to obtain a single cell suspension. The mononuclear splenocytes were isolated by differential centrifugation $(800 \times g$ for $40 \mathrm{~min}$ at room temperature [RT]) on a Ficoll-Hypaque cushion (Histopaque 1.077, Sigma, St. Louis, MO, USA). The cell suspension was washed twice in RPMI-1640 and resuspended to obtain $10^{7}$ cells $/ \mathrm{mL}$.

\subsection{Determination of parasite load index - LDU}

Following necropsy, fragments of spleen were collected and imprints prepared on two microscope slides. Slides were air-dried, fixed in methanol, stained with Giemsa, and examined under an optical microscopy to detect amastigote forms of Leishmania. Parasite densities were determined according to Stauber (1955) with some 
modifications, and expressed as Leishman Donovan Units (LDU index), which correspond the number of Leishmania amastigotes per 1000 nucleated cells. For control purposes, seven dogs that were not infected with CVL according to the IFAT assay were also submitted to parasitological examination.

\subsection{Immunophenotyping by flow cytometry}

Immunophenotyping analysis of splenocytes by flow cytometry was undertaken as described by Reis et al. (2005). Briefly, $1 \times 10^{7}$ cells $/ \mathrm{mL}$ of splenocytes were subjected to pre-fixation and erythrocyte lysis by the gentle addition of $13 \mathrm{~mL}$ lysis solution (FACS lysing solution, Becton Dickinson, Mountain View, CA, USA) followed by incubation for $10 \mathrm{~min}$ at RT. After centrifugation $(450 \times \mathrm{g}$ for $10 \mathrm{~min}$ at RT), the pellet was resuspended in $500 \mu \mathrm{L}$ phosphate buffered saline supplemented with $10 \%$ of fetal bovine serum.

In a 96-well U-bottom plate (Limbro Biomedicals, Inc., Aurora, OH, USA), $30 \mu \mathrm{L}$ of pre-fixed splenocyte suspension was incubated at RT for $30 \mathrm{~min}$, in the dark, in the presence of $30 \mu \mathrm{L}$ of anti-canine cell surface marker antibodies. Monoclonal antibodies (mAbs), which define canine cell phenotypes, including purified rat anti-dog CD5 (Rat-IgG2a, clone YKIX322.3), anti-dog CD4 (RatIgG2a, clone YKIX302.9), anti-dog CD8 (Rat-IgG1, clone YCATE55.9), anti-MHC-II (Rat-IgG2b, clone YKIX334.2), anti-CD45RA (Rat-IgG2b, clone YKIX753.22.2), and antiCD45RB (Rat-IgG2b, clone YKIX716.13) were used in an indirect immunofluorescence procedure. FITC-labeled mouse anti-human-CD21 (Mouse-IgG1, clone IOBla) was used in direct immunofluorescence procedures. Unlabeled mAbs used in this study were purchased from Serotec (Oxford, UK) and anti-CD21 from Immunotech Co. (Marselle, France).

During the immunophenotyping the cells were also incubated under the same conditions in the presence of $60 \mu \mathrm{L}$ of previously diluted FITC-conjugated sheep anti-rat IgG antibody. Before flow cytometric data collection and analysis, labeled cells were fixed for 30 min with $200 \mu \mathrm{L}$ of FACS fix solution (10.0 g/L paraformaldehyde; $10.2 \mathrm{~g} / \mathrm{L}$ sodium cacodylate, and $6.65 \mathrm{~g} / \mathrm{L}$ sodium chloride, $\mathrm{pH} 7.2$ ).

\subsection{Flow cytometry data storage and analysis}

Flow cytometric measurements were performed on a FACScan instrument (Becton Dickinson). The Cell-Quest software package was used in both data acquisition and analysis. A total of 10,000 events were acquired for each preparation. Canine whole-blood leucocytes were identified on the basis of their specific forward (FSC) and side (SSC) light-scatter properties as described by Reis et al. (2005). The results were expressed as the percentage of positive cells within the selected lymphocyte gate for $\mathrm{CD}^{+}$, $\mathrm{CD}^{+}, \mathrm{CD}^{+}$, and $\mathrm{CD} 21^{+}$cells. Semi-quantitative analyses were performed to evaluate differential expression of cell surface markers presenting a unimodal distribution $\left(\mathrm{MHC}-\mathrm{II}^{+}, \mathrm{CD} 45 \mathrm{RA}^{+}\right.$, and $\mathrm{CD} 45 \mathrm{RB}^{+}$), and the results were expressed as Mean Fluorescence Channel (MFC).

\subsection{Splenocytes proliferation assay}

The functional activity of splenocytes was determined using the lymphocyte proliferation assay. The cell suspension was adjusted to $10^{6}$ cells/mL of RPMI-1640 medium (Sigma) supplemented with $10 \%$ FCS, 100,000 U/L penicillin and $0.2 \mathrm{~g} / \mathrm{L}$ streptomycin. Two hundred microliters of the suspension was transferred into each well of a 96-well flatbottomed plate (Nunc, Naperville, IL, USA) containing $25 \mu \mathrm{l}$ of LSA ( $25 \mu \mathrm{g} / \mathrm{ml}$ ) obtained according Reis et al. (2006c), for the antigenic stimulus assays. The mitogenic lectin Concanavalin A (ConA, Sigma, USA) at $10 \mu \mathrm{g} / \mathrm{mL}$, and RPMI 1640 medium were used as controls. All samples were run in triplicate. The microplates were incubated at $37^{\circ} \mathrm{C}$ under a $5 \% \mathrm{CO}_{2}$ atmosphere for 3 days. Twenty hours before harvesting, $50 \mu \mathrm{L}$ of medium with ${ }^{3} \mathrm{H}$-thymidine $(5 \mu \mathrm{Ci} / \mathrm{mL})$ was added. The incorporation of ${ }^{3} \mathrm{H}$-thymidine was measured with a liquid scintillation counter (Tricarb CA 1600 , Packard BioScience Company, Meriden, CT, USA). The stimulation index was calculated as the ratio of counts per minute (CPM) in stimulated samples versus CPM in nonstimulated controls.

\subsection{Statistical analysis}

Statistical analysis was performed using GraphPad Prism 5.0. The normality of the data was assessed using the Kolmogorov-Smirnoff test. Parametric data were analysis by variance (ANOVA) followed by Tukey's test. Nonparametric data were analyzed by the Kruskal-Wallis test followed by Dunns' test. Student's $t$-test and the Mann-Whitney test were used to identify significant differences between the ratio of stimulated samples versus non-stimulated control cultures for parametric and nonparametric data, respectively. Correlation analysis was performed by Pearson's rank test in the case of parametric data and Spearman's rank test in the case of non-parametric data. In all cases, significance was defined at $p<0.05$.

\section{Results and discussion}

The role of the anti-Leishmania immune response underlying the susceptibility/resistance during CVL has been recognized in many ex vivo and in vitro investigations (Cardoso et al., 2007; Lage et al., 2007; Pinelli et al., 1994, 1995; Reis et al., 2006a). Recently, we demonstrated that immunoglobulin levels, as well as the parasite load are relevant hallmarks of distinct clinical status of CVL (Coura-Vital et al., 2011a; Reis et al., 2009, 2006c). To better understand the events underlying the compartmentalized immune response in the spleen, we performed a detailed analysis of clinical status of CVL and ex vivo and in vitro phenotypic features of the major splenocyte subsets $\left(\mathrm{CD}^{+}, \mathrm{CD}^{+}, \mathrm{CD}^{+}\right.$, $\mathrm{CD} 21^{+}$), and several activation markers ( MHC- $\mathrm{II}^{+}, \mathrm{CD}^{+} 5 \mathrm{RA}^{+}$, $\mathrm{CD}_{45} \mathrm{RB}^{+}$). The spleen is one of the tissues where the parasite replicates, and is an important organ responsible for adaptive immune response during CVL infection. Thus, the assessment of cellular immunophenotypic profile may improve our understanding of the relationship between the major events of disease progression with uncontrolled parasite replication in the splenic compartment. 

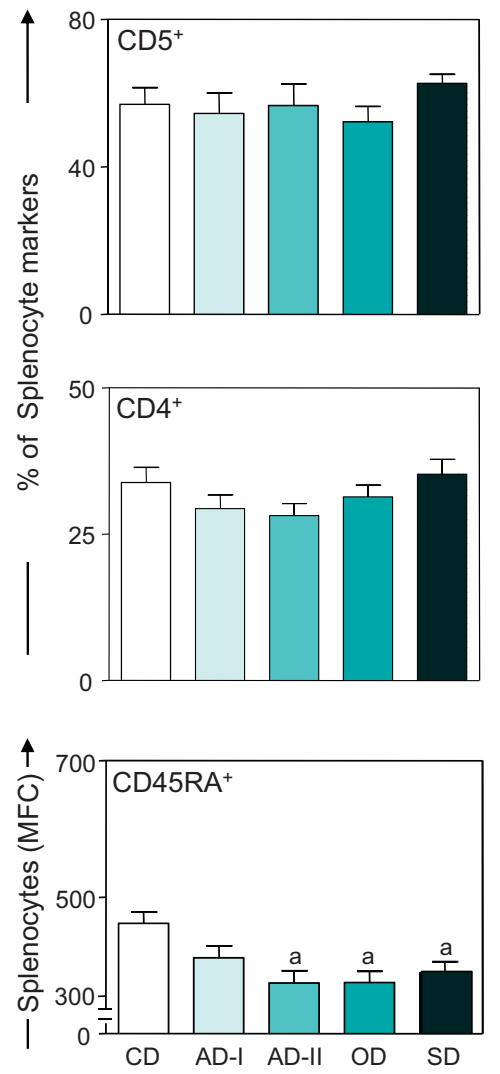
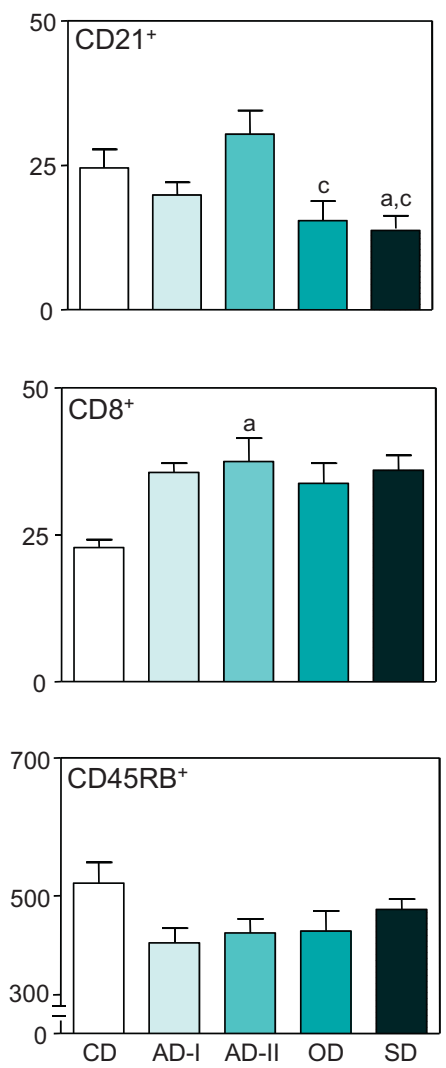

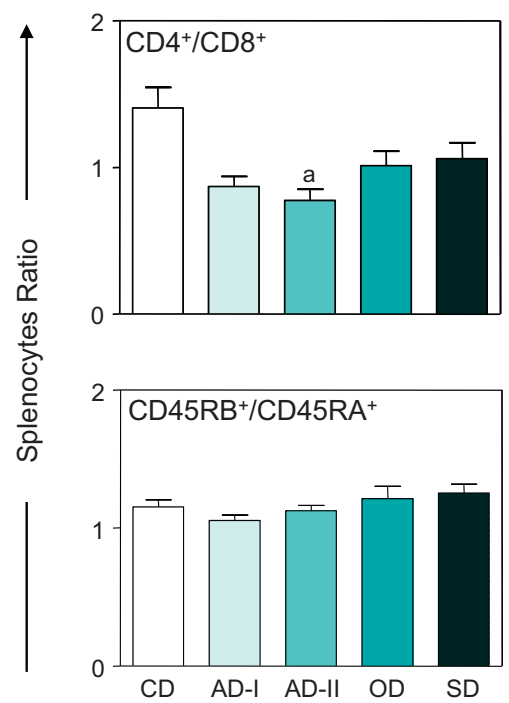

Clinical status

Fig. 1. Immunophenotypic profile of splenocytes in dogs naturally infected with Leishmania infantum categorized according to their clinical status as asymptomatic (AD), oligosymptomatic (OD), symptomatic (SD), and control (CD) groups. The results are expressed in bar graphs as mean percentage of $\mathrm{CD}^{+}, \mathrm{CD} 21^{+}, \mathrm{CD}^{+}, \mathrm{CD}^{+}$within gated lymphocytes, and $\mathrm{CD}^{+} / \mathrm{CD}^{+}$ratio plus standard deviation. The results of $\mathrm{CD}^{+} \mathrm{R} \mathrm{R}^{+}$and $\mathrm{CD} 45 \mathrm{RB}^{+}$are expressed as average of mean fluorescence channel (MFC), and CD45RB ${ }^{+} / C_{4} 45 R A^{+}$ratio plus standard deviation. Significant differences at $p<0.05$ are indicated by the letters $a$ and $c$ in comparison with the $\mathrm{CD}$ and $\mathrm{AD}-\mathrm{II}$ groups, respectively.

Studies have reported a decrease in the percentage of $\mathrm{B}$ cells $\left(\mathrm{CD} 21^{+}\right)$in peripheral blood of dogs naturally infected with $L$. infantum (Bourdoiseau et al., 1997; Reis et al., 2006b). In the present study, ex vivo immunophenotypic analysis of splenocytes also showed a reduction in the percentage of B lymphocytes $\left(\mathrm{CD} 21^{+}\right)$in the OD group as compared with AD-II, as well as in the SD group as compared with both $\mathrm{CD}$ and $\mathrm{AD}-\mathrm{II}$ (Fig. 1). The decrease of $B$ cells in dogs with active CVL is intriguing and unexpected, mainly because these animals exhibit intense polyclonal B cells, as evidenced by increased production of immunoglobulin of various classes and subclasses (Martinez-Moreno et al., 1995; Reis et al., 2006c; CouraVital et al., 2011a). Results obtained by Guerra et al. (2009), however, demonstrated no difference in the percentage of this lymphocyte subpopulation in the spleen when the animals were categorized by parasite load. Thus, we suggest that the decreased percentage of $\mathrm{B}$ cells $\left(\mathrm{CD} 21^{+}\right)$in peripheral blood (Reis et al., 2006b), and spleen as well from symptomatic dogs (SD) could reflect an involvement in the production of these cells in the bone marrow and a possible differentiation in plasma cells.
We also observed that $\mathrm{CD}^{+} \mathrm{T}$ cells reflect most accurately both the clinical status and the overall bone marrow parasite density, as increased levels of $\mathrm{CD}^{+}$circulating lymphocytes appeared as the major phenotypic feature of asymptomatic disease and dogs bearing a low parasite load (Reis et al., 2006b). In the present study, we did not observe alterations in $\mathrm{CD}^{+}$and $\mathrm{CD} 4^{+}$splenocytes in ex vivo experiments, whereas the AD-II group presented a high percentage of $\mathrm{CD}^{+} \mathrm{T}$ cells, and reduction in the $\mathrm{CD} 4^{+} / \mathrm{CD}^{+}$ ratio (Fig. 1). Coura-Vital et al. (2011a) observed similar results with higher $\mathrm{CD}^{+} \mathrm{T}$ cells, and lower $\mathrm{CD} 4 / \mathrm{CD} 8$ ratio in AD-II and SD groups in the peripheral blood compared with $C D$. In another study that evaluated the immunophenotyping of leukocytes from liver and spleen from naturally infected dogs showed that the splenic immune responses in both symptomatic and asymptomatic dogs were very similar (Sanchez et al., 2004). Guerra et al. (2009) showed in naturally infected dogs that, the increase frequency of $\mathrm{CD}^{+} \mathrm{T}$ cells in peripheral blood is strongly associated with low splenic parasitism. In our current study, we observed that low parasite load in spleen is found mainly in asymptomatic dogs (AD-I and AD-II) confirming 


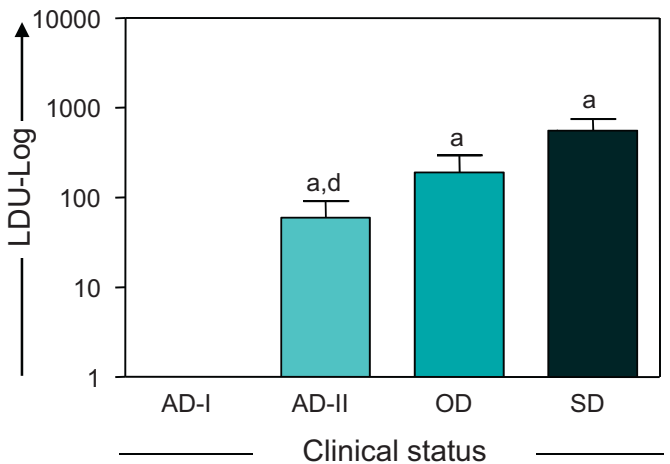

Fig. 2. Splenic parasite load in dogs naturally infected with Leishmania infantum categorized according to their clinical status as asymptomatic I (AD-I), asymptomatic II (AD-II), oligosymptomatic (OD) and symptomatic (SD) groups. The results are expressed in bar graphs as average of Leishman Donovan Units (LDU) in log scale plus standard deviation. Significant differences at $p<0.05$ are indicated by the letters $a$ and $d$ in comparison with the AD-I and SD groups, respectively.

this hypothesis. In fact, different studies have correlated the level of $\mathrm{CD}^{+} \mathrm{T}$ cells with protection during $L$. infantum infection (Pinelli, 1997; Reis et al., 2006b) or during the immunogenic response after vaccine administration against CVL (Giunchetti et al., 2007, 2008; Roatt et al., 2012). Thus, our data also suggest that $\mathrm{CD}^{+} \mathrm{T}$ cells in several compartments may control the parasite replication.

Furthermore, we evaluated the ex vivo $\mathrm{CD}_{45 \mathrm{RA}^{+}}$and $\mathrm{CD}_{45 \mathrm{RB}^{+}}$expression on splenocytes in different clinical groups. The results showed a decreased expression of $\mathrm{CD}_{45 \mathrm{RA}^{+}}$in the animals after seroconversion. However, no differences were observed for $C D 45 \mathrm{RB}^{+}$expression or the $\mathrm{CD}_{45 \mathrm{RB}}{ }^{+} / \mathrm{CD} 45 \mathrm{RA} \mathrm{A}^{+}$expression ratio among the groups (Fig. 1). Guerra et al. (2009) also observed decreased CD45RA ${ }^{+}$expression on splenocytes from animals infected with a moderate parasite load. These changes may be associated with the high splenic parasitism frequently noticed in dogs with high parasite load or exhibiting severe CVL clinical status.

Spleen parasite load was assessed using Giemsa-stained microscopy to determine the Leishman Donovan Units (LDU) (Fig. 2). Data analysis demonstrated a higher parasite load in SD, OD, and AD-II groups compared with AD-I dogs. Interestingly, we observed lower parasitism in AD-II than SD dogs in the splenic compartment (Fig. 2). Sanchez et al. (2004) suggested that the striking differences in parasite burden between AD and SD animals could be explained by a diminished infection or a more efficient control of the parasite replication in the former group, as we observed in our study.

To evaluate a Leishmania-specific cell-mediated immune response, splenocytes from dogs with CVL were stimulated by a mitogen (ConA) (Fig. 3A) or specific antigen (LSA) (Fig. 3B). Our results demonstrated that a lymphoproliferative response decreased in all seropositive dogs in comparison with the $C D$ group, when simulated with ConA or in all infected dogs after simulation with LSA. Furthermore, a correlation analysis between non-specific and LSA-specific lymphoproliferative response and clinical status revealed that severe CVL disease is correlated with a low proliferation index during the infection $(r=-0.3627$, $p=0.0113 ; r=-0.5263, p=0.0001$, respectively; Fig. $3 \mathrm{~A}$ and B). Similar results have been documented by other research groups (Cabral et al., 1998; Pinelli et al., 1994; Rodriguez-Cortes et al., 2007).

Moreover, it was conducted an analysis of the phenotypic features in the spleen compartment in the presence or absence of LSA stimulation (Fig. 4). We observed an increased percentage of $\mathrm{CD}^{+}$splenocytes in OD and SD as compared with $\mathrm{CD}$ in non-stimulated cultures. Moreover, the stimulated cultures with LSA antigens showed increased percentage of $\mathrm{CD}^{+} \mathrm{T}$ cells in $\mathrm{AD}-\mathrm{II}$ as compared with the $C D$ group or non-stimulated culture in the same group (Fig. 4A). Similarly, Pinelli et al. (1995) also observed that T lymphocytes from symptomatic dogs failed to proliferate after Leishmania antigen stimulation in vitro. On the other hand, these authors reported that asymptomatic dogs showed both $\mathrm{CD}^{+} \mathrm{T}$ and $\mathrm{CD} 8^{+} \mathrm{T}$ lymphocyte proliferation, and were capable of lysing infected macrophages.

We also investigated whether dogs with distinct clinical forms of CVL present alterations in the expression of activation markers (CD45RA ${ }^{+}, \mathrm{CD}_{4} 4 \mathrm{RB}^{+}$, and $\mathrm{MHC}-\mathrm{II}^{+}$) on splenocytes (Fig. 4B). The development of a T-cell mediated specific immune response requires Leishmania antigen presentation to Th cells together with major
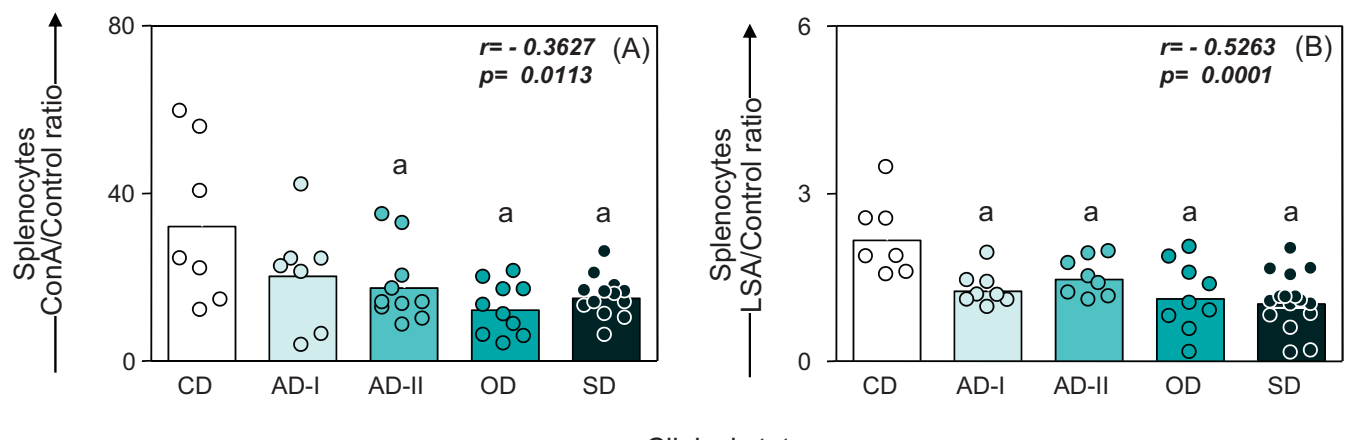

Clinical status

Fig. 3. Proliferative index of splenocytes in dogs naturally infected with Leishmania infantum categorized according to their clinical status as asymptomatic (AD), oligosymptomatic (OD), symptomatic (SD), and control (CD), after in vitro Concanavalin A (ConA) or L. infantum soluble antigen (LSA) stimulation. The results are expressed in bar graphs as scattering of individual values and mean ConA/Control ratio (A) or LSA/Control ratio (B). Correlations between proliferation indices and clinical status are also inserted, and Pearson's correlation coefficient $(r)$ and $p$-values are shown within graphs. 


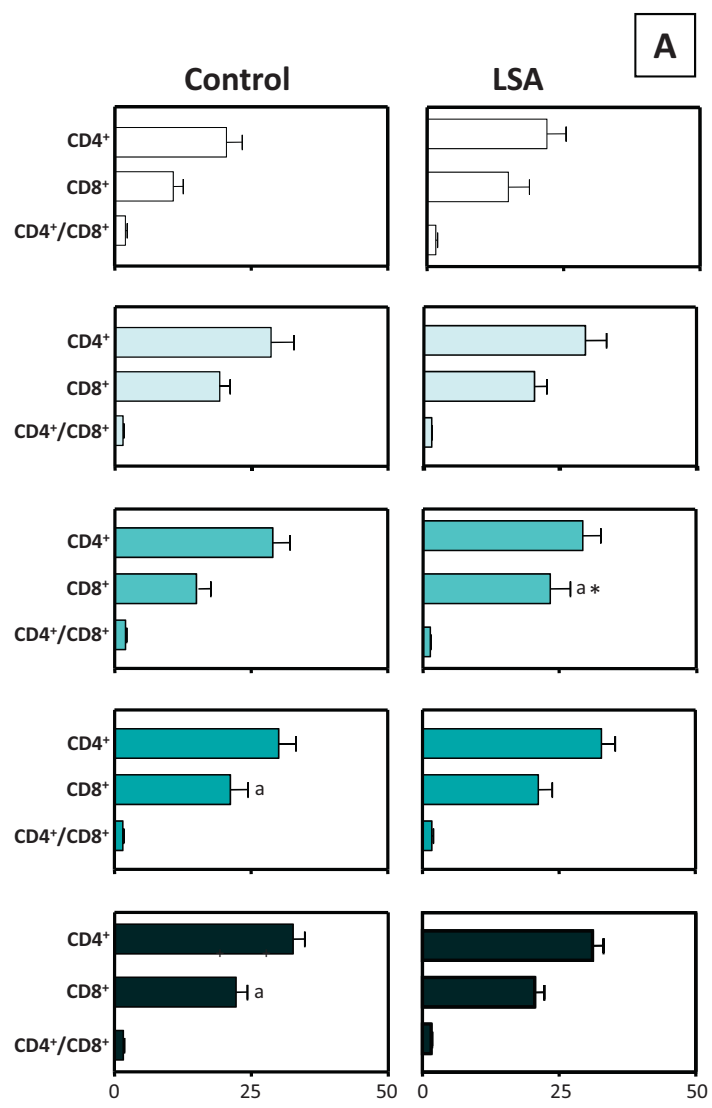

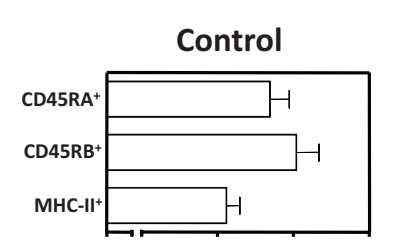
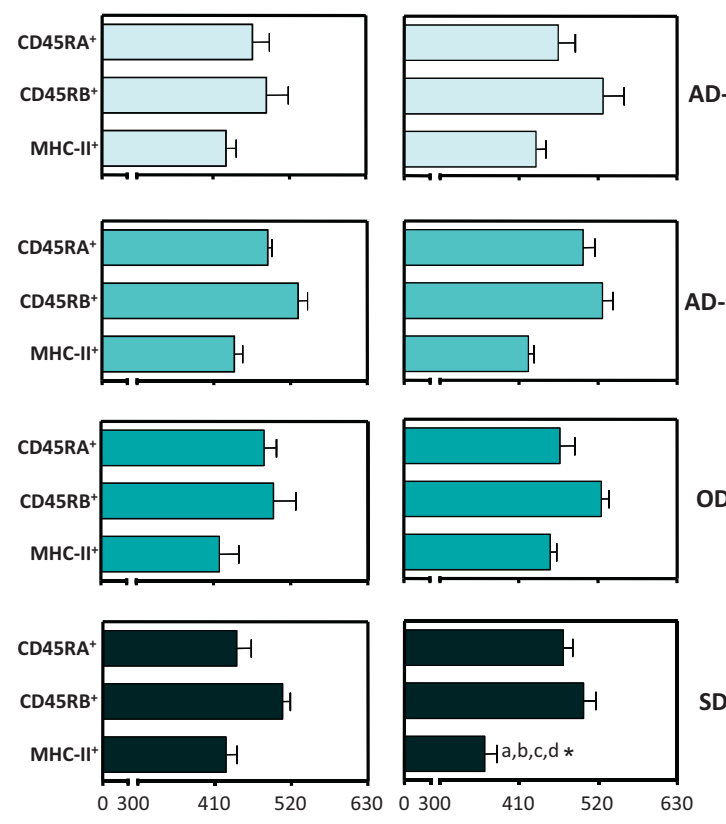

Fig. 4. Immunophenotypic profile and activation status of splenocytes in dogs naturally infected with Leishmania infantum categorized according to their clinical status as asymptomatic (AD), oligosymptomatic (OD), symptomatic (SD), and control (CD), after in vitro culture in the presence of medium (Control) or L. infantum soluble antigen (LSA) for $120 \mathrm{~h}$. The results are expressed in bar graphs as mean percentage of CD $4^{+}$and CD8 ${ }^{+}$within gated lymphocytes and $\mathrm{CD}^{+} / \mathrm{CD}^{+}$ratio plus standard deviation (A), and average of major histocompatibility complex (MHC-II ${ }^{+}$), $\mathrm{CD}^{2}$ RA ${ }^{+}$, and $\mathrm{CD} 45 \mathrm{RB}^{+}$as mean fluorescence channel (MFC) within gated splenocytes plus standard deviation (B). Significant differences at $p<0.05$ are indicated by the letters $a, b, c$, and $d$ in comparison with the CD, AD-I, AD-II, and OD groups, respectively. Significant differences at $p<0.05$ between control and $L$. infantum soluble antigen (LSA) cultures are indicated by asterisk.

histocompatibility complex (MHC) molecules. However, Leishmania can impair antigen presentation in several ways. Reis et al. (2006b) demonstrated enhanced MHC-II density as well as a higher CD45RB/CD45RA expression index in peripheral blood from asymptomatic dogs, which seems to represent a key element to control CVL morbidity. In the present study, we observed decreased MHC-II expression in splenocytes after LSA stimulation in SD group as compared with all groups or non-stimulated culture in the same group (Fig. 4B). Cobbold and Metcalfe (1994) performed pioneering investigations of MHC-II molecules in dogs. They observed that, in contrast with other species, the MHC-II of dogs is expressed constitutively in all circulating lymphocytes. Increased expression of MHC-II may reflect an antigenic priming-related immunological event. Based on this proposal, our findings suggest that symptomatic dogs displayed lower MHC-II expression, probably promoting more vulnerability to disease.

In conclusion, our findings highlighted the complexity of cellular immunological events related to the natural infection of dogs by L. infantum. Our results showed that the role of $\mathrm{CD}^{+} \mathrm{T}$ splenocytes seems to be key for an effective immunological response, a hallmark of asymptomatic CVL, whereas the pronounced loss of MHC-II expression on splenocytes upon specific- antigen stimulation is a biomarker of symptomatic CVL.

\section{Acknowledgements}

The authors express their appreciation of the hard work carried out by the kennel staff at the Universidade Federal de Ouro Preto, and Universidade Federal de Minas Gerais, and for their special dedication during the execution of this study. We also thank the people from Fundação Nacional da Saúde, Ministério da Saúde, Distrito Regional de Belo Horizonte, Minas Gerais, for their special dedication to this work. The authors also thank the Program for Technological Development in Tools for Health-PDTIS-FIOCRUZ, CEBIO, Universidade Federal de Minas Gerais, Instituto René Rachou (IRR/FIOCRUZ Minas), Rede Mineira de Bioterismo, FAPEMIG (Fundação de Amparo à Pesquisa do Estado de Minas Gerais). A.B.R., A.T.C., R.C.G., R.C.O., and O.A.M.F. thank Conselho Nacional de Desenvolvimento Científico e 
Tecnológico (CNPq), and W.C.V. thank CAPES/PNPD for fellowships.

\section{References}

Baneth, G., Koutinas, A.F., Solano-Gallego, L., Bourdeau, P., Ferrer, L., 2008. Canine leishmaniosis - new concepts and insights on an expanding zoonosis: part one. Trends Parasitol. 24, 324-330.

Boggiatto, P.M., Ramer-Tait, A.E., Metz, K., Kramer, E.E., Gibson-Corley, K., Mullin, K., Hostetter, J.M., Gallup, J.M., Jones, D.E., Petersen, C.A., 2010. Immunologic indicators of clinical progression during canine Leishmania infantum infection. Clin. Vaccine Immunol. 17, 267-273.

Bourdoiseau, G., Bonnefont, C., Magnol, J.P., Saint-Andre, I., Chabanne, L. 1997. Lymphocyte subset abnormalities in canine leishmaniasis. Vet. Immunol. Immunopathol. 56, 345-351.

Cabral, M., O’Grady, J., Alexander, J., 1992. Demonstration of Leishmania specific cell mediated and humoral immunity in asymptomatic dogs. Parasite Immunol. 14, 531-539.

Cabral, M., O'Grady, J.E., Gomes, S., Sousa, J.C., Thompson, H., Alexander, J., 1998. The immunology of canine leishmaniosis: strong evidence for a developing disease spectrum from asymptomatic dogs. Vet. Parasitol. $76,173-180$

Cardoso, L., Schallig, H.D., Cordeiro-da-Silva, A., Cabral, M., Alunda, J.M. Rodrigues, M., 2007. Anti-Leishmania humoral and cellular immune responses in naturally infected symptomatic and asymptomatic dogs. Vet. Immunol. Immunopathol. 117, 35-41.

Cobbold, S., Metcalfe, S., 1994. Monoclonal antibodies that define canine homologues of human CD antigens: summary of the First International Canine Leukocyte Antigen Workshop (CLAW). Tissue Antigens 43, 137-154

Coura-Vital, W., Marques, M.J., Giunchetti, R.C., Teixeira-Carvalho, A., Moreira, N.D., Vitoriano-Souza, J., Vieira, P.M., Carneiro, C.M. Correa-Oliveira, R., Martins-Filho, O.A., Carneiro, M., Reis, A.B., 2011a. Humoral and cellular immune responses in dogs with inapparent natural Leishmania infantum infection. Vet. J. 190, e43-e47.

Coura-Vital, W., Marques, M.J., Veloso, V.M., Roatt, B.M., Aguiar-Soares, R.D., Reis, L.E., Braga, S.L., Morais, M.H., Reis, A.B., Carneiro, M., 2011b. Prevalence and factors associated with Leishmania infantum infection of dogs from an urban area of Brazil as identified by molecular methods. PLoS Negl. Trop. Dis. 5, e1291.

Degrave, W., Fernandes, O., Campbell, D., Bozza, M., Lopes, U., 1994. Use of molecular probes and PCR for detection and typing of Leishmania-a mini-review. Mem. Inst. Oswaldo Cruz. 89, 463-469.

Desjeux, P., 2004. Leishmaniasis: current situation and new perspectives. Comp. Immunol. Microbiol. Infect. Dis. 27, 305-318.

Giunchetti, R.C., Correa-Oliveira, R., Martins-Filho, O.A., TeixeiraCarvalho, A., Roatt, B.M., de Oliveira Aguiar-Soares, R.D., de Souza, J.V., das Dores Moreira, N., Malaquias, L.C., Mota e Castro, L.L., de Lana, M., Reis, A.B., 2007. Immunogenicity of a killed Leishmania vaccine with saponin adjuvant in dogs. Vaccine 25, 7674-7686

Giunchetti, R.C., Reis, A.B., da Silveira-Lemos, D., Martins-Filho, O.A., Correa-Oliveira, R., Bethony, J., Vale, A.M., da Silva Quetz, J. Bueno, L.L., Franca-Silva, J.C., Nascimento, E., Mayrink, W., Fujiwara, R.T., 2008. Antigenicity of a whole parasite vaccine as promising candidate against canine leishmaniasis. Res. Vet. Sci. 85, 106-112.

Goto, H., Prianti, M.G., 2009. Immunoactivation and immunopathogeny during active visceral leishmaniasis. Rev. Inst. Med. Trop. Sao Paulo $51,241-246$.

Guerra, L.L., Teixeira-Carvalho, A., Giunchetti, R.C., Martins-Filho, O.A., Reis, A.B., Corrêa-Oliveira, R., 2009. Evaluation of the influence of tissue parasite density on hematological and phenotypic cellular parameters of circulating leukocytes and splenocytes during ongoing canine visceral leishmaniasis. Parasitol Res. 104, 611-622.

Kaye, P.M., Svensson, M., Ato, M., Maroof, A., Polley, R., Stager, S., Zubairi, S., Engwerda, C.R., 2004. The immunopathology of experimental visceral leishmaniasis. Immunol. Rev. 201, 239-253.

Lage, R.S., Oliveira, G.C., Busek, S.U., Guerra, L.L., Giunchetti, R.C., CorreaOliveira, R., Reis, A.B., 2007. Analysis of the cytokine profile in spleen cells from dogs naturally infected by Leishmania chagasi. Vet. Immunol. Immunopathol. 115, 135-145.

Mancianti, F., Gramiccia, M., Gradoni, L., Pieri, S., 1988. Studies on canine leishmaniasis control. Evolution of infection of different clinical forms of canine leishmaniasis following antimonial treatment. Trans. R. Soc. Trop. Med. Hyg. 82, 566-567.
Martinez-Moreno, A., Moreno, T., Martinez-Moreno, F.J., Acosta, I., Hernandez, S., 1995. Humoral and cell-mediated immunity in natural and experimental canine leishmaniasis. Vet. Immunol. Immunopathol. 48 , 209-220.

Nylen, S., Sacks, D., 2007. Interleukin-10 and the pathogenesis of human visceral leishmaniasis. Trends Immunol. 28, 378-384.

Pinelli, E., 1997. Cytokines in canine visceral leishmaniasis. In: Virgil, E.C.J., Schijns, V.E.C.J., Horzinek, M.C. (Eds.), Cytokines in Veterinary Medicine. Cab International, United Kingdom, pp. 217-224.

Pinelli, E., Boog, C.J., Rutten, V.P., van Dijk, B., Bernadina, W.E., Ruitenberg, E.J., 1994. A canine CD8+ cytotoxic T-cell line specific for Leishmania infantum-infected macrophages. Tissue Antigens 43, 189-192.

Pinelli, E., Gonzalo, R.M., Boog, C.J., Rutten, V.P., Gebhard, D., del Real, G. Ruitenberg, E.J., 1995. Leishmania infantum-specific T cell lines derived from asymptomatic dogs that lyse infected macrophages in a major histocompatibility complex-restricted manner. Eur. J. Immunol. 25 1594-1600.

Pinelli, E., Rutten, V.P., Bruysters, M., Moore, P.F., Ruitenberg, E.J., 1999. Compensation for decreased expression of B7 molecules on Leishmania infantum-infected canine macrophages results in restoration of parasite-specific T-cell proliferation and gamma interferon production. Infect. Immun. 67, 237-243.

Reis, A.B., Carneiro, C.M., Carvalho, M.G., Teixeira-Carvalho, A., Giunchetti, R.C., Mayrink, W., Genaro, O., Correa-Oliveira, R., Martins-Filho, O.A 2005. Establishment of a microplate assay for flow cytometric assessment and it is use for the evaluation of age-related phenotypic changes in canine whole blood leukocytes. Vet. Immunol. Immunopathol. 103, $173-185$

Reis, A.B., Martins-Filho, O.A., Teixeira-Carvalho, A., Carvalho, M.G. Mayrink, W., Franca-Silva, J.C., Giunchetti, R.C., Genaro, O., CorreaOliveira, R., 2006a. Parasite density and impaired biochemi$\mathrm{cal} /$ hematological status are associated with severe clinical aspects of canine visceral leishmaniasis. Res. Vet. Sci. 81, 68-75.

Reis, A.B., Martins-Filho, O.A., Teixeira-Carvalho, A., Giunchetti, R.C. Carneiro, C.M., Mayrink, W., Tafuri, W.L., Correa-Oliveira, R., 2009. Systemic and compartmentalized immune response in canine visceral leishmaniasis. Vet. Immunol. Immunopathol. 128, 87-95.

Reis, A.B., Teixeira-Carvalho, A., Giunchetti, R.C., Guerra, L.L., Carvalho, M.G., Mayrink, W., Genaro, O., Correa-Oliveira, R., Martins-Filho, O.A. 2006b. Phenotypic features of circulating leucocytes as immunological markers for clinical status and bone marrow parasite density in dogs naturally infected by Leishmania chagasi. Clin. Exp. Immunol. 146 303-311

Reis, A.B., Teixeira-Carvalho, A., Vale, A.M., Marques, M.J., Giunchetti, R.C., Mayrink, W., Guerra, L.L., Andrade, R.A., Correa-Oliveira, R., MartinsFilho, O.A., 2006c. Isotype patterns of immunoglobulins: hallmarks for clinical status and tissue parasite density in Brazilian dogs naturally infected by Leishmania (Leishmania) chagasi. Vet. Immunol. Immunopathol. 112, 102-116.

Roatt, B.M., Aguiar-Soares, R.D., Vitoriano-Souza, J., Coura-Vital, W., Braga, S.L., Correa-Oliveira, R., Martins-Filho, O.A., Teixeira-Carvalho, A., de Lana, M., Gontijo, N.F., Marques, M.J., Giunchetti, R.C., Reis, A.B., 2012. Performance of LBSap vaccine after intradermal challenge with $L$ infantum and saliva of Lu. longipalpis: immunogenicity and parasitological evaluation. PLoS One 7, e49780.

Rodriguez-Cortes, A., Fernandez-Bellon, H., Ramis, A., Ferrer, L., Alberola, J., Solano-Gallego, L., 2007. Leishmania-specific isotype levels and their relationship with specific cell-mediated immunity parameters in canine leishmaniasis. Vet. Immunol. Immunopathol. 116, 190-198.

Sanchez, M.A., Diaz, N.L., Zerpa, O., Negron, E., Convit, J., Tapia, F.J., 2004 Organ-specific immunity in canine visceral leishmaniasis: analysis of symptomatic and asymptomatic dogs naturally infected with Leishmania chagasi. Am. J. Trop. Med. Hyg. 70, 618-624.

Seixas Duarte, M.I., Tuon, F.F., Pagliari, C., Kauffman, M.R., Brasil, R.A., 2008. Human visceral leishmaniasis expresses Th1 pattern in situ liver lesions. J. Infect. 57, 332-337.

Stanley, A.C., Engwerda, C.R., 2007. Balancing immunity and pathology in visceral leishmaniasis. Immunol. Cell Biol. 85, 138-147.

Stauber, L.A., 1955. Leishmaniasis in the hamster. In: Cole, W.H. (Ed.) Some Physiological Aspects and Consequence of Parasitism. Rugers University Press, New Brunswick, NJ, pp. 77-90.

Strauss-Ayali, D., Baneth, G., Shor, S., Okano, F., Jaffe, C.L., 2005 Interleukin-12 augments a Th1-type immune response manifested as lymphocyte proliferation and interferon gamma production in Leish mania infantum-infected dogs. Int. J. Parasitol. 35, 63-73.

Volpini, A.C., Passos, V.M., Oliveira, G.C., Romanha, A.J., 2004. PCR-RFLP to identify Leishmania (Viannia) braziliensis and L. (Leishmania) amazonensis causing American cutaneous leishmaniasis. Acta Trop. 90 $31-37$ 